TRANSACTIONS OF THE

AMERICAN MATHEMATICAL SOCIETY

Volume 358, Number 11, November 2006, Pages 4919-4933

S 0002-9947(06)03993-6

Article electronically published on April 11, 2006

\title{
SEMIFREE SYMPLECTIC CIRCLE ACTIONS ON 4-ORBIFOLDS
}

\author{
L. GODINHO
}

\begin{abstract}
A theorem of Tolman and Weitsman states that all symplectic semifree circle actions with isolated fixed points on compact symplectic manifolds must be Hamiltonian and have the same equivariant cohomology and Chern classes of $\left(\mathbb{C} P^{1}\right)^{n}$ equipped with the standard diagonal circle action. In this paper, we show that the situation is much different when we consider compact symplectic orbifolds. Focusing on 4-orbifolds with isolated cone singularities, we show that such actions, besides being Hamiltonian, can now be obtained from either $S^{2} \times S^{2}$ or a weighted projective space, or a quotient of one of these spaces by a finite cyclic group, by a sequence of special weighted blow-ups at fixed points. In particular, they can have any number of fixed points.
\end{abstract}

\section{INTRODUCTION}

Tolman and Weitsman showed in [T-W] that any semifree circle action (that is, free outside the fixed point set) with isolated fixed points on a compact symplectic manifold must be Hamiltonian. Moreover, $S^{2} \times S^{2}$ with the standard diagonal circle action is, up to symplectomorphism, the only 4-dimensional symplectic manifold with such an action. In higher dimensions, the only known example is still the product of spheres with the standard diagonal circle action, and the classical manifolds invariants (cohomology and Chern classes), as well as the equivariant cohomology of these spaces, must agree with those of $\left(\mathbb{C} P^{1}\right)^{n}$ (cf. [T-W] for details).

The situation is much different when we consider semifree circle actions on orbifolds. In this paper, we focus on such actions on 4-dimensional symplectic orbifolds with isolated cone singularities (that is, for which the orbifold structure groups of their orbifold points are cyclic groups acting diagonally). Also, we prove that, besides being Hamiltonian, they can now be obtained from either $S^{2} \times S^{2}$ with the standard diagonal action, or the weighted projective space $\mathbb{C} P^{2}(a, b, a+b)$ with the $S^{1}$-action defined by

$$
e^{\theta i} \cdot\left[z_{1}: z_{2}: z_{3}\right]=\left[e^{q \theta i} z_{1}: e^{p \theta i} z_{2}: e^{(p+q) \theta i} z_{3}\right],
$$

where $p$ and $q$ are integers satisfying $p a-q b=1$, or a quotient of one of these $S^{1}$-spaces by a cyclic group $\mathbb{Z}_{k}$ acting as a subgroup of $S^{1}$, by a sequence of special weighted blow-ups at fixed points. We describe the two basic examples in Section 3 , the weighted blow-ups in Section 4, and, in Section 15, we obtain the following results.

Received by the editors September 21, 2004.

2000 Mathematics Subject Classification. Primary 53D20.

This research was partially supported by FCT through program POCTI/FEDER and grant POCTI/MAT/57888/2004, and by Fundação Calouste Gulbenkian. 
Theorem 1.1. A symplectic circle action on a compact symplectic 4-dimensional orbifold is Hamiltonian iff it has a non-empty fixed-point set.

Theorem 1.2. Every semifree Hamiltonian circle action on a compact symplectic 4-dimensional orbifold with isolated cone singularities can be obtained by a sequence of equivariant weighted blow-ups at fixed points from either:

1. a product of two spheres, $S^{2} \times S^{2}$, with the standard diagonal circle action, or

2. a weighted projective space $\mathbb{C} P^{2}(a, b, a+b)$ with the $S^{1}$-action defined by $e^{\theta i} \cdot\left[z_{1}: z_{2}: z_{3}\right]=\left[e^{q \theta i} z_{1}: e^{p \theta i} z_{2}: e^{(p+q) \theta i} z_{3}\right]$, where $p$ and $q$ are integers satisfying $p a-q b=1$, or

3. a quotient of one of the above $S^{1}$-spaces by a cyclic group $\mathbb{Z}_{k}$ acting as a subgroup of $S^{1}$, equipped with the induced $S^{1} \cong S^{1} / \mathbb{Z}_{k}$ action,

with a symplectic form that comes from a Kähler form. Moreover, when the blownup point has index 2, the sum of the orders of the orbifold structure group of the fixed points on the exceptional divisor is equal to the order of the orbifold structure group of the blown-up point. If, on the other hand, the blown-up point is a fixed point of index 0 or 4, then the absolute value of the difference between the orders of the orbifold structure groups of the fixed points on the exceptional divisor is equal to the order of the orbifold structure group of the blown-up point. In particular, these orbifolds are toric.

Theorem 1.1 is proved using the Atiyah-Bott and Berline-Vergne ( $[\mathrm{A}-\mathrm{B}], \mathrm{B}-\mathrm{V}]$ ) localization formula for equivariant cohomology (generalized to orbifolds by Meinrenken $[\mathrm{M}]$ ).

If we do not assume that the orbifold singularities are isolated, more examples can occur. The argument in Theorem 1.2 is generalized in Section 6 to show that any such space can be obtained from an $S^{1}$-orbifold with the same fixed-point data (that is, number of fixed points, orders of their orbifold structure groups and their isotropy weights) as

1. a product of two "footballs" $\left(\mathbb{C} P^{1}(a, b) \times \mathbb{C} P^{1}(c, d)\right.$ with $a, b, c, d$ pairwise relatively prime) equipped with a diagonal circle action

$$
e^{\theta i} \cdot\left(\left[z_{1}: z_{2}\right],\left[w_{1}: w_{2}\right]\right)=\left(\left[e^{q \theta i} z_{1}: e^{p \theta i} z_{2}\right],\left[e^{s \theta i} w_{1}: e^{r \theta i} w_{2}\right]\right),
$$

where $p, q, r, s$ are integers satisfying $p a-q b=1$ and $r c-s d=1$, or

2. a weighted projective space $\mathbb{C} P^{2}(a b c, b d e, a d(c+e))$ for which there exist integers $p, q, r$ satisfying $p(d e)-q(a c)=1$ and $p d+q a=r b$, equipped with the circle action given by

$$
e^{\theta i} \cdot\left[z_{1}: z_{2}: z_{3}\right]=\left[e^{p \theta i} z_{1}: e^{q \theta i} z_{2}: e^{r \theta i} z_{3}\right] \text {, or }
$$

3. a quotient of one of the above $S^{1}$-spaces by a cyclic group $\mathbb{Z}_{k}$ acting as a subgroup of $S^{1}$, equipped with the induced $S^{1} \cong S^{1} / \mathbb{Z}_{k}$ action.

Remark. In the isolated case, we perform a sequence of weighted blow-ups to obtain a manifold and use the classification of $S^{1}$ actions on 4-manifolds to conclude that any semifree action can be obtained (by a sequence of equivariant weighted blowups at fixed points) from an orbifold equivariantly diffeomorphic to one of the above examples with isolated singularities. In the general case, however, we may not be able to obtain a manifold from this sequence of blow ups, so we can only conclude that such actions can be obtained (by a sequence of weighted blow-ups) from a space with the same fixed-point data as in examples $\mathbf{1}, \mathbf{2}$ or $\mathbf{3}$. 


\section{ORBIFOLDS AND GROUP ACTIONS}

In this section we review some background material on orbifolds and group actions.

2.1. Orbifolds. An orbifold $M$ is a singular manifold whose singularities are locally isomorphic to quotient singularities of the form $\mathbb{R}^{n} / \Gamma$, where $\Gamma$ is a finite subgroup of $G L(n, \mathbb{R})$. An orbifold chart for $M$ is a triple $(U, V, \Gamma)$ consisting of an open subset $U$ of $M$, a finite group $\Gamma \subset G L(n, \mathbb{R})$, an open subset $V$ of $\mathbb{R}^{n}$ and a homeomorphism $U=V / \Gamma$. An orbifold structure on the paracompact Hausdorff space $M$ is then a collection of orbifold charts covering $M$, subject to appropriate compatibility conditions. These, in particular, ensure that, on each connected component, the generic stabilizers of the $\Gamma_{i}$-actions are isomorphic. For each singularity $p \in M$, there is a finite subgroup $\Gamma_{p}$ of $G L(n, \mathbb{R})$, unique up to conjugation, such that open neighborhoods of $p$ in $M$ are homeomorphic to neighborhoods of the origin in $\mathbb{R}^{n} / \Gamma$. A singular point is called an orbifold point and $\Gamma_{p}$ its orbifold structure group.

The definitions of vector fields, differential forms, metrics, group actions, etc. naturally extend to orbifolds. For instance, a symplectic orbifold is an orbifold equipped with a closed non-degenerate 2 -form $\omega$. Given any orbifold $M$, fiber orbibundles $\pi: E \rightarrow M$ are defined by $\Gamma$-equivariant fiber bundles $Z \rightarrow E_{V} \rightarrow V$ in orbifold charts $(U, \Gamma, V)$, together with suitable compatibility conditions. The fibers $\pi^{-1}(p)$ are not, in general, diffeomorphic to $Z$, but to some quotient of $Z$ by the action of the orbifold structure group $\Gamma_{p}$.

All orbifolds we will consider in this paper will have a presentation of the form $M=X / T$, where $X$ is a manifold and $T$ is a torus acting in an effective, locally free fashion on $X$. All orbifold points $p$ will be assumed to be cone singularities, that is, to have a cyclic group $\mathbb{Z}_{k}$ as orbifold structure group, acting diagonally on $\mathbb{R}^{n}$. Moreover, all 4-orbifolds that we will consider will be generically manifolds.

Just as any line bundle over a sphere is isomorphic to some line bundle $\mathcal{O}(k)$ of Chern class $k \in \mathbb{Z}$ defined by $S^{3} \times \mathbb{C} / \sim$, where $\left(z_{1}, z_{2}, w\right) \sim\left(\lambda z_{1}, \lambda z_{2}, \lambda^{k} w\right)$ for $\lambda \in$ $S^{1}$, any line orbi-bundle over an orbifold "sphere" $\mathbb{C} P^{1}(p, q)($ with $\left.\operatorname{gcd}(p, q)=1)\right)$ is isomorphic to some $\mathcal{O}_{p, q}(k / r)$ (where $k$ and $r$ are relatively prime and $r>0$ ), defined by $S^{3} \times \mathbb{C} / \sim$, where $\left(z_{1}, z_{2}, w\right) \sim\left(\lambda^{r p} z_{1}, \lambda^{r q} z_{2}, \lambda^{k} w\right)$ for $\lambda \in S^{1}$ (cf. [R] for details). Note that, if $k>0, \mathcal{O}_{p, q}(k / r)$ is isomorphic to the normal orbi-bundle of $\mathbb{C} P^{1}(r p, r q) \cong \mathbb{C} P^{1}(p, q)$ inside $\mathbb{C} P^{2}(r q, r p, k)$, so we will denote $\mathcal{O}_{p, q}(k / r)$ by $\mathcal{O}_{r p, r q}(k)$.

2.2. Group actions. Let $(M, \omega)$ be a symplectic orbifold, and $G$ a compact, connected Lie group acting smoothly on $M$. Since $G$ is connected, the components of the fixed-point set $M^{G}$ are suborbifolds of $M$. Moreover, if $G$ is abelian, the normal orbi-bundle $\nu_{F}$ to each fixed-point component $F$ is even dimensional, and admits an invariant Hermitean structure. If $M$ is oriented, any choice of such a Hermitean structure defines an orientation in $F$. The definition of equivariant differential forms, $\mathcal{A}_{G}(M)$ (that is, polynomial $G$-equivariant mappings $\alpha$ from $\mathfrak{g}$ to the space of differential forms in $M$ ), also extends naturally to orbifolds equipped with a group action, as well as the equivariant differential $d_{G}: \mathcal{A}_{G}(M) \rightarrow \mathcal{A}_{G}(M)$ defined as

$$
d_{G}(\alpha)(\xi)=d \alpha(\xi)-2 \pi i \iota\left(\xi_{M}\right) \alpha(\xi)
$$


where $\xi_{M}$ is the fundamental vector field corresponding to $\xi$. An example of an equivariantly closed form is the equivariant Euler class $e\left(\nu_{F}\right)$ of the normal orbibundle $\nu_{F}$. Supposing $G$ is abelian, and using the splitting principle if necessary, we can assume, without loss of generality, that $\nu_{F}$ splits into a direct sum of line orbi-bundles $L_{i}$ with first Chern classes $c_{1}\left(L_{i}\right)$, and (rational) orbi-weights $\lambda_{i}$, and we have

$$
e\left(\nu_{F}\right)=\prod_{i}\left(c_{1}\left(L_{i}\right)+\lambda_{i}\right)
$$

Let us now assume that $M$ is compact, connected and oriented, and consider the integration mapping $\int: \mathcal{A}_{G}(M) \rightarrow \mathcal{A}_{G}(M)$ and $\iota_{F}: F \rightarrow M$, the embeddings of the fixed-point orbifolds. The Atiyah-Bott and Berline-Vergne localization formula ([A-B], B-V]) was generalized to orbifolds by Meinrenken [M]:

Proposition 2.1 (Localization formula for orbifolds). Suppose $G$ is abelian, and let $\alpha \in \mathcal{A}_{G}(M)$ be $d_{G}$-closed. Then,

$$
\frac{1}{d_{M}} \int_{M} \alpha=\sum_{F} \frac{1}{d_{F}} \int_{F} \frac{\iota_{F}^{*} \alpha}{e\left(\nu_{F}\right)},
$$

where, for a connected orbifold $X, d_{X}$ is the order of the orbifold structure group of a generic point of $X$, and the sum is over the fixed-point orbifolds.

2.3. Circle actions. Let us now consider the case where $G=S^{1}$ acts in a Hamiltonian fashion on an orbifold $M$ with cone singularities. Most of the basic definitions of Morse theory on orbifolds are analogous to their manifold counterparts (cf. $[\mathrm{L}-\mathrm{T}]$ ). In particular, given a fixed point $F \in M$, there is a stable (or unstable) orbifold formed by the points which converge to $F$ under the flow of - grad $H$ (or $\operatorname{grad} H$ ), where $\operatorname{grad} H$ is the gradient of the Hamiltonian function with respect to a compatible Riemannian metric. Given the local form of $H$ and of its gradient near $F$, the closures of the stable and unstable orbifolds of $F$ have a cone singularity at $F$. If $M$ is 4-dimensional and $F$ is a fixed point of index 2, the closure of the stable (or unstable) orbifold is a sphere having a cone singularity at $F$ but which, in theory, could have other types of singularity at the other pole. However, if this other pole is another fixed point $F^{\prime}$ of index 2, this sphere also has a cone singularity at $F^{\prime}$ since it is then the unstable (or stable) orbifold of $F^{\prime}$. If, in addition, the components where $H$ takes its maximum (or minimum) are isolated points, then the stable (or unstable) orbifolds of fixed points of index 2 having these points as poles also have cone singularities at these poles (see $[\mathrm{A}-\mathrm{H}]$ for a detailed explanation of the manifold case). We conclude that, if all fixed points are isolated, they are poles of gradient orbifold spheres which are either teardrops or footballs (that is, weighted projective spaces or their quotients by a finite cyclic group).

Let us now assume that we have a semifree circle action on an orbifold with cone singularities and let $F$ be an isolated fixed point. A neighborhood of $F$ is modeled by the quotient $\mathbb{C}^{2} / \mathbb{Z}_{N}$ for an action of $\mathbb{Z}_{N}$ given by

$$
\xi_{N} \cdot(z, w)=\left(\xi_{N}^{m_{1}} z, \xi_{N}^{m_{2}} w\right),
$$

with $\left|m_{1}\right|,\left|m_{2}\right| \leq N$ and $\left(m_{1}, m_{2}\right)=1$. The circle action on this neighborhood will be given by

$$
e^{i \theta} \cdot(z, w)=\left(e^{i p \theta / N} z, e^{i q \theta / N} w\right)
$$


for some integers $p$ and $q$ and, since we are assuming that it is semifree, $p$ must divide $\left(m_{1}, N\right)$, and $q$ must divide $\left(m_{2}, N\right)$. Hence, in order to have a well-defined semifree action on this neighborhood, we must be able to choose $m_{1}$ and $m_{2}$ dividing $N$ and then $p= \pm m_{1}$ and $q= \pm m_{2}$. Consequently, the weights of the isotropy representation of $S^{1}$ on $T_{F} M$ are $\pm m_{1} / N$ and $\pm m_{2} / N$, and $\left|m_{1}\right|,\left|m_{2}\right|$, when different from 1, are the orders of the orbifold structure group of a generic point in two gradient "spheres" having $F$ as a pole. Note that, as we are assuming that our 4-orbifolds are generically manifolds, $\left|m_{1}\right| \neq\left|m_{2}\right|$ unless they are both equal to 1 , in which case $F$ is an isolated orbifold point (and the weights are $\pm 1 / N$ ). In this case, the action of $Z_{N}$ implies that, on $\mathbb{C}^{2} / \mathbb{Z}_{N}$,

$$
(z, w) \sim\left(\xi_{N} z, \xi_{N}^{ \pm 1} w\right) \sim\left(\xi_{N}^{k} z, \xi_{N}^{ \pm k} w\right),
$$

for any integer $k, 1 \leq k \leq N$, relatively prime with $N$, and $\xi_{N}$ a primitive $N$-th root of unity.

\section{BASIC EXAMPLES}

1. When $M$ is a 4-manifold, the only possible semifree circle action is the standard diagonal action on the product of two spheres $S^{2} \times S^{2}$ (cf. [T-W], [A] and $[\mathrm{K}]$ ). The circle acts by rotating each sphere while fixing the poles. There are four fixed points, $(n, n),(n, s),(s, n)$ and $(s, s)$, where $n$ and $s$ are the north and south poles of the sphere.

2. We will now consider a weighted projective space $\mathbb{C} P^{2}(a, b, a+b)$ where $a$ and $b$ are positive integers. This space is defined as the complex quotient $\left(\mathbb{C}^{3} \backslash\{0\}\right) / \mathbb{C}^{*}$ for an action of $\mathbb{C}^{*}=\mathbb{C} \backslash\{0\}$ given by

$$
\left(z_{1}, z_{2}, z_{3}\right) \sim\left(\lambda^{a} z_{1}, \lambda^{b} z_{2}, \lambda^{a+b} z_{3}\right), \lambda \in \mathbb{C}^{*},
$$

and can also be obtained by symplectic reduction. If $a, b$ and $a+b$ are pairwise relatively prime, this compact symplectic orbifold has three isolated orbifold singularities with orbifold structure groups isomorphic to $\mathbb{Z}_{a}, \mathbb{Z}_{b}$ and $\mathbb{Z}_{a+b}$. Let us now consider integers $p, q$ (given by the extended Euclidean algorithm) for which

$$
p a-q b=1,
$$

and the circle action on $\mathbb{C} P^{2}(a, b, a+b)$ given by

$$
e^{\theta i} \cdot\left[z_{1}: z_{2}: z_{3}\right]=\left[e^{q \theta i} z_{1}: e^{p \theta i} z_{2}: e^{(p+q) \theta i} z_{3}\right] .
$$

This action has three isolated fixed points $F_{1}=[1: 0: 0], F_{2}=[0: 1: 0]$ and $F_{3}=[0: 0: 1]$. A coordinate system centered at $F_{1}$ is given by

$$
(z, w) \in \mathbb{C}^{2} \longmapsto[1: z: w] .
$$

In this coordinate system $(z, w) \sim\left(\xi_{a} z, \xi_{a} w\right)$, where $\xi_{a}$ is a primitive $a$-th root of unity. The action of $S^{1}$ on this coordinate system is given by

$$
\begin{aligned}
& e^{\theta i} \cdot[1: z: w]=\left[e^{q i \theta}: e^{p i \theta} z: e^{(p+q) \theta i} w\right] \\
& =\left[1: e^{i(p a-q b) / a) \theta} z: e^{i(((p+q) a-q(a+b)) / a) \theta} w\right]=\left[1: e^{i / a \theta} z: e^{i / a \theta} w\right],
\end{aligned}
$$

implying that the weights of the isotropy representation of $S^{1}$ on $T_{F_{1}} M$ are both $1 / a$. Similarly, we can conclude that the weights on $T_{F_{2}} M$ are both $-1 / b$ and on $T_{F_{3}} M$ are $\pm 1 /(a+b)$.

We will see in Section 5 that all examples of semifree circle actions on orbifolds with isolated cone singularities can be obtained by a sequence of weighted blow-ups 
either from the above two examples or from a quotient of one of these spaces by a finite cyclic group. There are, however, more examples with non-isolated cone singularities, as can be seen in Section 6 .

\section{Weighted BLOW-UP}

In this section we will consider two special cases of weighted blow-up:

I. Let $F$ be an isolated cone singularity of orbifold structure group $\mathbb{Z}_{N}$. Let us assume that a neighborhood of $F$ is modeled by the quotient $\mathbb{C}^{2} / \mathbb{Z}_{N}$ for an action of $Z_{N}$ on $\mathbb{C}^{2}$ given by

$$
\xi_{N} \cdot(z, w)=\left(\xi_{N}^{k} z, \xi_{N}^{-k} w\right),
$$

where $\xi_{N}$ is a primitive $N$-th root of unity and $k$ is an integer $1 \leq k<N$ relatively prime with $N$. Moreover, consider the Hamiltonian circle action on $\mathbb{C}^{2}$ given by

$$
e^{i \theta} \cdot(z, w)=\left(e^{i a \theta} z, e^{i(N-a) \theta} w\right)
$$

with Hamiltonian function given by $\tilde{H}=\frac{1}{4}\left(a|z|^{2}+(N-a)|w|^{2}\right)$ for some integer $1 \leq a<N$. This action descends to a circle action on the quotient $\mathbb{C}^{2} / \mathbb{Z}_{N}$ having only one critical point (of index 0 ) at the origin. We can remove a neighborhood of this point of the form $H^{-1}([0, \epsilon))$, where $H$ is the Hamiltonian function of this new action, and collapse the boundary of the resulting orbifold along the orbits of the circle action. The exceptional divisor of this weighted blow-up is a weighted projective space $\mathbb{C} P^{1}(a, N-a)$.

If we have a circle action on $M$, it will descend to the weighted blow-up. In particular, the semifree action given, on a neighborhood of $F$, by

$$
e^{i \theta} \cdot(z, w)=\left(e^{i \theta / N} z, e^{-i \theta / N} w\right)
$$

will descend to a new semifree action on the the blown-up orbifold. Indeed, the exceptional divisor $\mathbb{C} P^{1}(a, N-a)$ will be an invariant orbifold $S^{2}$ where the circle action can be written in homogeneous coordinates as

$$
\begin{aligned}
{\left[e^{i \theta / N} z: e^{-i \theta / N} w\right] } & =\left[z: e^{-i \theta(1 / N+(N-a) /(N a))} w\right]=\left[z: e^{-i \theta / a} w\right] \\
& =\left[e^{i \theta(1 / N+a /(N(N-a)))} z: w\right]=\left[e^{i \theta /(N-a)} z: w\right],
\end{aligned}
$$

and so the isotropy weights at the new fixed points are $-1 / a$ and $1 /(N-a)$.

II. Let $F$ again be an isolated cone singularity with orbifold structure group $\mathbb{Z}_{N}$ but let us assume now that a neighborhood of $F$ is modeled by $\mathbb{C}^{2} / \mathbb{Z}_{N}$ for an action of $\mathbb{Z}_{N}$ on $\mathbb{C}^{2}$ given by

$$
\xi_{N} \cdot(z, w)=\left(\xi_{N}^{k} z, \xi_{N}^{k} w\right), 1 \leq k<N,
$$

where $k$ is an integer $1<k \leq N$, relatively prime with $N$, and $\xi_{N}$ is a primitive $N$-th root of unity. Let us consider the Hamiltonian circle action on $\mathbb{C}^{2}$ given by

$$
e^{i \theta} \cdot(z, w)=\left(e^{i a \theta} z, e^{i(N+a) \theta} w\right)
$$

for some integer $a \geq 1$. This action descends to the quotient $\mathbb{C}^{2} / \mathbb{Z}_{N}$, and we can again remove a neighborhood of $F$ of the form $H^{-1}([0, \varepsilon))$, where $H$ is the Hamiltonian function of the induced action on the quotient, and collapse the boundary of the resulting orbifold along the orbits of the circle action. The exceptional divisor obtained will now be a weighted projective space $\mathbb{C} P^{1}(a, N+a)$.

The semifree circle actions on $M$ given, on a neighborhood of $F$, by

$$
e^{i \theta} \cdot(z, w)=\left(e^{ \pm i \theta / N} z, e^{ \pm \theta / N} w\right),
$$


will also descend to semifree circle actions on the blown-up orbifold. Indeed, the actions on the exceptional divisor can now be written in homogeneous coordinates as

$$
\begin{aligned}
{\left[e^{ \pm i \theta / N} z: e^{ \pm i \theta / N} w\right] } & =\left[z: e^{ \pm i \theta(1 / N-(N+a) /(N a))} w\right]=\left[z: e^{\mp i \theta / a} w\right] \\
& =\left[e^{ \pm i \theta(1 / N-a /(N(N+a)))} z: w\right]=\left[e^{ \pm i \theta /(N+a)} z: w\right]
\end{aligned}
$$

and so the isotropy weights at the new fixed points are $\mp 1 / a$ and $\pm 1 /(N+a)$, respectively.

\section{Proof of the MAIN THEOREMS}

We begin this section with the proof of Theorem 1.1

Theorem 1.1. A symplectic circle action on a compact symplectic 4-dimensional orbifold is Hamiltonian iff it has a non-empty fixed-point set.

Proof. Let us assume that $M^{S^{1}} \neq \emptyset$. To show that any such action must be Hamiltonian, we first deform the symplectic form to a rational invariant symplectic form and we choose a compatible invariant almost complex structure. Even though this a.c.s. is not unique, the weights at every fixed point are well defined. For dimensional reasons, every fixed point of index 2 must be isolated, implying that none of the weights on their normal orbi-bundles can be zero. Since we now have an invariant rational symplectic form, the circle action will have a circle-valued moment map (the argument follows the one for the manifold case described in MD]). If this map had no local minima or maxima, then, applying the equivariant integration formula of Proposition 2.1 to $\alpha=1$, yields

$$
0=\frac{1}{d} \int_{M} 1=\sum_{F \in M^{S^{1}}} \frac{1}{d_{F}} \frac{1}{e\left(\nu_{F}\right)}
$$

where $d_{F}$ denotes the order of the orbifold structure group of the fixed point $F$, $e\left(\nu_{F}\right)$ is the equivariant Euler class of the tangent bundle at $F$, and the sum is over all fixed points $F$. Since we are assuming that all fixed points have index 2 , applying (2.1) we get

$$
e\left(\nu_{F}\right)=-k_{F}\left(\frac{u}{d_{F}}\right)^{2}
$$

for some positive integers $k_{F}$ and so, by (5.1), $\sum_{F \in M^{S^{1}}} k_{F}^{-1} d_{F}=0$, which is impossible. We conclude that the circle-valued moment map has a local minimum, and so it must lift to a real-valued moment map, implying that any symplectic circle action on $M$ must be Hamiltonian. Moreover, the real-valued moment map is a Morse function with only even-index critical points, so our orbifold is simply connected (for Morse theory on orbifolds see e.g. [L-T]).

Conversely, if the action is Hamiltonian, it must have fixed points (the critical points of the corresponding Hamiltonian function).

Before proving our main result we need the following lemmas.

Lemma 1. Let $S^{1}$ act on a weighted projective space $\mathbb{C} P^{1}(p, q)$ by rotation, while fixing the north and south poles (orbifold singularities). Suppose that the action lifts to a complex line orbi-bundle $\mathcal{O}_{r p, r q}(k)$, where $k$ and $r$ are relatively prime and $r>0$. Then $S^{1}$ acts linearly on the fibers over the north and south poles and, 
denoting by $m_{+}$and $m_{-}$the weights for these actions, $m_{+}-m_{-}=n\left(\frac{k}{p q r}\right)$ for some integer $n$. Moreover, if the action is semifree, $n=-1$.

Proof. Let $S^{1}$ act on the weighted projective space $\mathbb{C} P^{1}(p, q)$ by rotation

$$
e^{i \theta} \cdot\left[z_{0}: z_{1}\right]=\left[e^{i a \theta} z_{0}: e^{i b \theta} z_{1}\right], a, b \in \mathbb{Z} \backslash\{0\},
$$

having $[1: 0]$ and $[0: 1]$ as fixed points. For $k \in \mathbb{Z}$ and $p, q, r \in \mathbb{Z}^{+}$with $(k, r)=1$, the line orbi-bundle $\mathcal{O}_{r p, r q}(k)$ over $\mathbb{C} P^{1}(p, q)$ is defined as

$$
\mathcal{O}_{p, q}(k)=\left(S^{3} \times \mathbb{C}\right) / \sim,
$$

where $\left(z_{0}, z_{1}, w\right) \sim\left(\lambda^{r p} z_{0}, \lambda^{r q} z_{1}, \lambda^{k} w\right)$, for $\lambda \in S^{1}$. Let $\left[z_{0}: z_{1}: w\right]$ denote the equivalence class of $\left(z_{0}, z_{1}, w\right)$. Then, any lifted $S^{1}$-action over $\mathcal{O}_{r p, r q}(k)$ is of the form

$$
e^{i \theta} \cdot\left[z_{0}: z_{1}: w\right]=\left[e^{i a \theta} z_{0}: e^{i b \theta} z_{1}: e^{i c \theta} w\right]
$$

for some integer $c$. On the fiber over $[1: 0]$, we have

$$
e^{i \theta} \cdot[1: 0: w]=\left[e^{i a \theta}: 0: e^{i c \theta} w\right]=\left[1: 0: e^{i(c-(a k) /(r p)) \theta} w\right]
$$

and, over $[0: 1]$, we have

$$
e^{i \theta} \cdot[0: 1: w]=\left[0: e^{i b \theta}: e^{i c \theta} w\right]=\left[0: 1: e^{i(c-(b k) /(r q)) \theta} w\right],
$$

implying that $m_{+}=c-(b k) /(r q)$ and $m_{-}=c-(a k) /(r p)$, and so $m_{+}-m_{-}=$ $(a q-b p) k /(p q r)$. Moreover, if the action on $\mathbb{C} P^{1}(p, q) \cong \mathbb{C} P^{1}(r p, r q)$ is semifree, then $a-(b p) / q=-1 / q$ and $b-(a q) / p=1 / p$, implying that $a q-b p=-1$, and the result follows.

Lemma 2. Let $S_{(n p, n q)}^{2}$ be the orbifold "sphere" $\mathbb{C} P^{1}(p, q) / \mathbb{Z}_{n}$, for a $\mathbb{Z}_{n}$-action given by

$$
\xi_{n} \cdot\left[z_{0}: z_{1}\right]=\left[\xi_{n}^{a} z_{0}: \xi_{n}^{b} z_{1}\right],
$$

where $a, b$ are integers satisfying $b p-a q=1$ and $\xi_{n}$ is a primitive $n$-th root of unity. Let the circle act by rotation on $S_{(n p, n q)}^{2}$, while fixing the north and south poles. If the action lifts to a semifree $S^{1}$-action on a complex line orbi-bundle over $S_{(n p, n q)}^{2}$ with isolated orbifold points, then this orbi-bundle is of the form $\mathcal{O}_{p, q}(k) / \mathbb{Z}_{n}$ with $|k|=|q \pm p|$, for $\mathbb{Z}_{n}$-actions given by

$$
\xi_{n} \cdot\left[z_{0}: z_{1}: w\right]=\left[\xi_{n}^{a} z_{0}: \xi_{n}^{b} z_{1}: \xi_{n}^{-(b \pm a)} w\right]
$$

when $k=-(q \pm p)$, and

$$
\xi_{n} \cdot\left[z_{0}: z_{1}: w\right]=\left[\xi_{n}^{a} z_{0}: \xi_{n}^{b} z_{1}: \xi_{n}^{b \pm a} w\right],
$$

when $k=q \pm p$. Moreover, the weights $m_{+}$and $m_{-}$of the circle action over the north and south poles satisfy $m_{+}-m_{-}=-k /(p q)$.

Proof. A complex line orbi-bundle over $S_{(n p, n q)}^{2}$ with isolated orbifold points is given by

$$
\frac{D^{2+} \times \mathbb{C}}{\mathbb{Z}_{n p}} \cup_{\alpha} \frac{D^{2-} \times \mathbb{C}}{\mathbb{Z}_{n q}}
$$


where $D^{2+}$ and $D^{2-}$ are two (positively and negatively oriented) disks, $\mathbb{Z}_{n p}$ and $\mathbb{Z}_{n q}$ act on $D^{2 \pm} \times \mathbb{C}$ respectively by

$$
\xi_{n p} \cdot(z, w)=\left(\xi_{n p} z, \xi_{n p}^{k_{1}} w\right) \text { and } \xi_{n q} \cdot(z, w)=\left(\xi_{n q} z, \xi_{n q}^{k_{2}} w\right),
$$

where $\xi_{n p}$ and $\xi_{n q}$ are primitive $n p$ - and $n q$-th roots of unity, and the gluing map $\alpha:\left(\partial D^{2} \times \mathbb{C}\right) / \mathbb{Z}_{n p} \rightarrow\left(\partial D^{2} \times \mathbb{C}\right) / \mathbb{Z}_{n q}$ is given by

$$
\alpha\left(e^{\theta i}, w\right)=\left(e^{-\frac{p}{q} \theta i}, e^{-\frac{k_{1} q+k_{2} p}{q} \theta i} w\right) .
$$

Since this orbi-bundle admits a semifree $S^{1}$-action, $k_{1}, k_{2}= \pm 1$. Let us assume that $k_{1}=-1$ and $k_{2}=\mp 1$ (the case $k_{1}=1$ is similar), implying that $\alpha\left(e^{\theta i}, w\right)=$ $\left(e^{-\frac{p}{q} \theta i}, e^{\frac{q \pm p}{q} \theta i} w\right)$.

Let us now see that this orbi-bundle is the quotient $\mathcal{O}_{p, q}(-(q \pm p)) / \mathbb{Z}_{n}$ for the above $\mathbb{Z}_{n}$-action. A coordinate system centered at $[1: 0: 0]$ on $\mathcal{O}_{p, q}(-(q \pm p)) / \mathbb{Z}_{n}$ is given by $[z, w] \in \mathbb{C}^{2} / \mathbb{Z}_{n p} \rightarrow[1: z: w]$, where

$$
(z, w) \sim\left(\xi_{n}^{b} \xi_{n}^{-a q / p} z, \xi_{n}^{-(b \pm a)} \xi_{n}^{a(q \pm p) / p} w\right) \sim\left(\xi_{n p} z, \xi_{n p}^{-1} w\right),
$$

for $\xi_{n p}$ a primitive $n p$-th root of unity. Similarly, a coordinate system centered at $[0: 1: 0]$ is given by $[z, w] \in \mathbb{C}^{2} / \mathbb{Z}_{n q} \rightarrow[z: 1: w]$, where

$$
(z, w) \sim\left(\xi_{n}^{a} \xi_{n}^{-b p / q} z, \xi_{n}^{-(b \pm a)} \xi_{n}^{b(q \pm p) / q}\right) \sim\left(\xi_{n q} z, \xi_{n q}^{\mp 1} w\right),
$$

for $\xi_{n q}$ a primitive $n q$-th root of unity, and the result follows (note that $m_{+}=$ $-k_{1} /(n p)$ and $\left.m_{-}=k_{2} /(n q)\right)$.

Using these lemmas we have:

Theorem 1.2. Every semifree Hamiltonian circle action on a compact symplectic 4-dimensional orbifold with isolated cone singularities can be obtained by a sequence of equivariant weighted blow-ups at fixed points from either:

1. a product of two spheres, $S^{2} \times S^{2}$, with the standard diagonal circle action, or

2. a weighted projective space $\mathbb{C} P^{2}(a, b, a+b)$ with the $S^{1}$-action defined by $e^{\theta i} \cdot\left[z_{1}: z_{2}: z_{3}\right]=\left[e^{q \theta i} z_{1}: e^{p \theta i} z_{2}: e^{(p+q) \theta i} z_{3}\right]$, where $p$ and $q$ are integers satisfying $p a-q b=1$, or

3. a quotient of one of the above $S^{1}$-spaces by a cyclic group $\mathbb{Z}_{k}$ acting as a subgroup of $S^{1}$, equipped with the induced $S^{1} \cong S^{1} / \mathbb{Z}_{k}$ action,

with a symplectic form that comes from a Kähler form. Moreover, when the blownup point has index 2 , the sum of the orders of the orbifold structure group of the fixed points on the exceptional divisor is equal to the order of the orbifold structure group of the blown-up point. If, on the other hand, the blown-up point is a fixed point of index 0 or 4, then the absolute value of the difference between the orders of the orbifold structure groups of the fixed points on the exceptional divisor is equal to the order of the orbifold structure group of the blown-up point. In particular, these orbifolds are toric.

Proof. Knowing that the action is Hamiltonian (implying that we have a maximum and a minimum), Proposition 2.1 applied again to $\alpha=1$, yields

$$
d_{0}+d_{4}=\sum_{F} d_{F},
$$


where $d_{0}$ and $d_{4}$ are the orders of the orbifold structure groups of the fixed points of index 0 and 4 respectively, and the sum is taken over all fixed points of index 2 . Here we used the fact that, since the action is semifree and the orbifold singularities are isolated, the isotropy weights on the normal orbi-bundle of a fixed point with orbifold structure group $\mathbb{Z}_{d}$ are $\pm 1 / d$.

We will now see that we can blow down every gradient orbifold sphere passing through two index-2 fixed points. Indeed, if $d_{N}$ and $d_{S}$ are the orders of the orbifold structure groups of the north and south poles of one of these "spheres", we have that $m_{+}$and $m_{-}$, the isotropy weights over the north and south poles, are $1 / d_{N}$ and $-1 / d_{S}$, respectively. Hence, $m_{+}-m_{-}=\left(d_{S}+d_{N}\right) /\left(d_{S} d_{N}\right)$ and so, if $\left(d_{S}, d_{N}\right)=1$, by Lemma 1 a neighborhood of this "sphere" can be modeled by a neighborhood of the zero section on the orbi-bundle $\mathcal{O}_{d_{S}, d_{N}}\left(-\left(d_{S}+d_{N}\right)\right)$, implying that we can blow it down by the opposite process of the one described in Section 4 I. Indeed, we can remove an equivariant neighborhood of the exceptional "sphere" and replace it by a "ball" of the form $D^{2} / \mathbb{Z}_{d_{S}+d_{N}}$ of an appropriate radius, getting a new symplectic orbifold with a new semifree circle action. Here the action of $\mathbb{Z}_{d_{S}+d_{N}}$ on $D^{2}$ is given by

$$
\xi_{d_{S}+d_{N}} \cdot(z, w)=\left(\xi_{d_{S}+d_{N}}^{d_{S}} z, \xi_{d_{S}+d_{N}}^{d_{N}} w\right)=\left(\xi_{d_{S}+d_{N}}^{d_{S}} z, \xi_{d_{S}+d_{N}}^{-d_{S}} w\right) .
$$

If, on the other hand, $\left(d_{S}, d_{N}\right)=k \neq 1$, by Lemma 2, a neighborhood of this "sphere" can be modeled by a neighborhood of the zero section on the orbi-bundle $\mathcal{O}_{d_{S} / k, d_{N} / k}\left(-\left(d_{S}+d_{N}\right) / k\right) / \mathbb{Z}_{k}$, implying that we can remove an equivariant neighborhood of the gradient "sphere" and replace it by a "ball" of the form $D^{2} / \mathbb{Z}_{d_{S}+d_{N}}$ of an appropriate radius where the action of $\mathbb{Z}_{d_{S}+d_{N}}$ on $D^{2}$ is given by

$$
\xi_{d_{S}+d_{N}} \cdot(z, w)=\left(\xi_{d_{S}+d_{N}} z, \xi_{d_{S}+d_{N}}^{-1} w\right) .
$$

This "ball" can be identified with a neighborhood of the orbifold point of order $d_{S}+d_{N}$ inside the quotient $\mathbb{C} P^{2}\left(d_{S} / k, d_{N} / k,\left(d_{S}+d_{N}\right) / k\right) / \mathbb{Z}_{k}$, for the $\mathbb{Z}_{k}$-action on the weighted projective space given by

$$
\xi_{k} \cdot\left[z_{0}: z_{1}: z_{2}\right]=\left[\xi_{k}^{a} z_{0}: \xi_{k}^{b} z_{1}: \xi_{k}^{a+b} z_{2}\right],
$$

where $a$ and $b$ are integers satisfying $b d_{S}-a d_{N}=k$. This weighted blow-down is then the quotient by the action of $\mathbb{Z}_{k}$ of the opposite process of the one described in Section $4 \mathbf{I}$, and it is easy to verify that we still get a semifree circle action on the blown-down orbifold.

After we blow down all these "spheres", every fixed point of index 2 will be directly connected to both the fixed point of index 0 and the fixed point of index 4 by gradient "spheres". Let $d$ denote the orbifold structure group of one of these index-2 fixed points, and $d_{0}$ and $d_{4}$ denote the orders of the orbifold structure groups of the fixed points of index 0 and 4 , respectively. Then, the difference between the weights at the north and south poles for the two orbifold spheres that go through this fixed point of index 2 are

$$
\frac{1}{d}-\frac{1}{d_{0}}=\frac{d_{0}-d}{d d_{0}} \text { and }-\frac{1}{d_{4}}-\left(-\frac{1}{d}\right)=\frac{d_{4}-d}{d d_{4}} .
$$

Consequently, by Lemmas 1 and 2 above, for each fixed point of index 2 for which $d_{0}-d>0$ or $d_{4}-d>0$, we can still blow down one of the above "spheres" and have a semifree action on the resulting orbifold. Indeed, we can use the opposite process of the one described in Section 4.II or its quotient by the action of $\mathbb{Z}_{k}$ given by Lemma 2, where now $k=\left(d, d_{0}\right)$ or $\left(d, d_{4}\right)$, removing an equivariant neighborhood 
of the exceptional "sphere" and replacing it by a "ball" of the form $D^{2} / \mathbb{Z}_{d_{0}-d}$ or of the form $D^{2} / \mathbb{Z}_{d_{4}-d}$, for actions of $\mathbb{Z}_{d_{0}-d}$ and $\mathbb{Z}_{d_{4}-d}$ on $\mathbb{C}^{2}$ given respectively by

$$
\xi_{d_{0}-d} \cdot(z, w)=\left(\xi_{d_{0}-d} z, \xi_{d_{0}-d} w\right) \quad \text { and } \quad \xi_{d_{4}-d} \cdot(z, w)=\left(\xi_{d_{4}-d} z, \xi_{d_{4}-d} w\right)
$$

where $\xi_{d_{0}-d}$ and $\xi_{d_{4}-d}$ are primitive $\left(d_{0}-d\right)$ - and $\left(d_{4}-d\right)$-th roots of unity.

Note that there is at least one fixed point of index 2 for which $d \geq d_{0}$ and $d \geq d_{4}$ since, if that were not the case, we would obtain (by blowing down) an orbifold with only two fixed points (of index 0 and 4), which is impossible by (5.2). Hence, we just have to consider the case where all index-2 fixed points satisfy $d \geq d_{0}$ and $d \geq d_{4}$. If there were three or more of these points, then the sum of the orders of the corresponding orbifold structure groups would be greater than $d_{0}+d_{4}$, violating (5.2). If there were two of these points, then $d_{0}=d_{4}$, and the orders of their orbifold structure groups would have to be equal to $d_{0}$ (that is $d_{i}=d_{0}=d_{4}$ ). If $d_{0}=1$, we are in the manifold case, implying that $M$ is equivariantly diffeomorphic to $S^{2} \times S^{2}$ equipped with the standard diagonal action. Otherwise, $M$ has the same fixed-point data (that is, number of fixed points, orders of their orbifold structure groups and their isotropy weights) as the quotient $\left(S^{2} \times S^{2}\right) / \mathbb{Z}_{d_{0}}$, for the standard diagonal action of $\mathbb{Z}_{d_{0}}$, equipped with the $S^{1} \cong S^{1} / \mathbb{Z}_{d_{0}}$ action induced by the standard diagonal circle action on the product of two spheres.

Finally, if we only have one fixed point of index 2, then its orbifold structure group has order equal to $d_{0}+d_{4}$. If $\left(d_{0}, d_{4}\right)=1$, then we have the same fixed-point data as in example 2 of Section 3 with $a=d_{0}$ and $b=d_{N}$. If, on the other hand, $\left(d_{0}, d_{4}\right)=k \neq 1$, we have the same fixed-point data as the quotient of one of those spaces, $\mathbb{C} P^{2}\left(d_{0} / k, d_{4} / k,\left(d_{0}+d_{4}\right) / k\right)$, by the action of $\mathbb{Z}_{k}$ given by

$$
\xi_{k} \cdot\left[z_{0}: z_{1}: z_{2}\right]=\left[\xi_{k}^{q} z_{0}: \xi_{k}^{p} z_{1}: \xi_{k}^{p+q} z_{2}\right],
$$

where $p$ and $q$ are integers satisfying $p d_{0}-q d_{4}=k$, equipped with the $S^{1} \cong$ $S^{1} / \mathbb{Z}_{k}$ action induced by the corresponding semifree circle action on the weighted projective space.

Consequently, after we blow down all possible gradient "spheres", we obtain one of the cases depicted in Figure 1, where the fixed points are represented by dots, labeled by the orders of their orbifold structure groups ( $a$ and $b$ are now arbitrary positive integers).

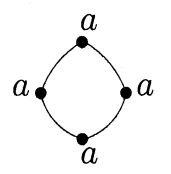

$(A)$

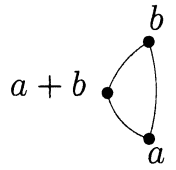

$(B)$

Figure 1.

Hence, to finish our proof, we just have to show that an orbifold $M$ equipped with a semifree circle action with the same fixed-point data as one of the above examples is, in fact, equivariantly diffeomorphic to that space. Let us first consider an orbifold $M$ with the fixed-point data of Figure $1(B)$ and let $M^{\prime}$ be either the weighted projective space $M^{\prime}=\mathbb{C} P^{2}(a, b, a+b)$ (when $(a, b)=1$ ), or the quotient $M^{\prime}=\mathbb{C} P^{2}(a / k, b / k,(a+b) / k) / \mathbb{Z}_{k}$ (when $\left.(a, b)=k \neq 1\right)$. Then we perform, on both $M$ and the corresponding space, $M^{\prime}$, a sequence of weighted blow-ups (like 


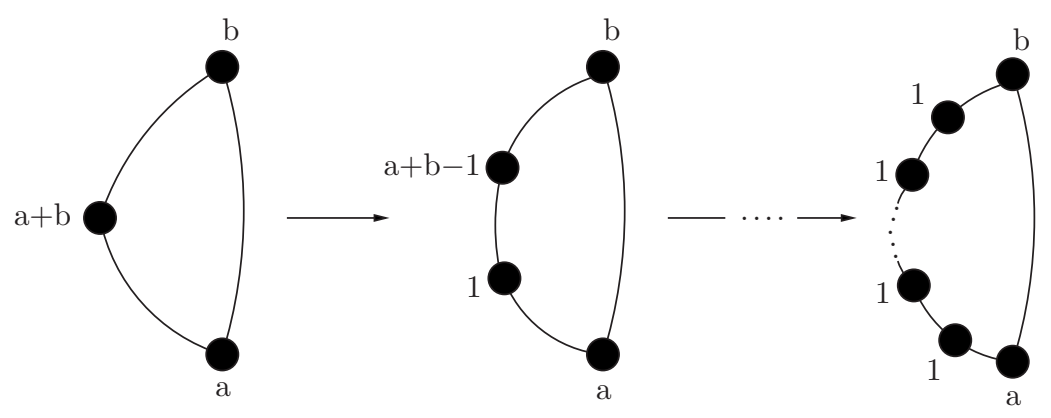

FiguRE 2. Blowing up fixed points of index 2

the ones described in Section 4), blowing up fixed points until we obtain a circle action on a smooth manifold. Then, we use the results of Audin and Karshon in A] and $[\mathrm{K}]$ to show that these manifolds are equivariantly diffeomorphic, implying that $M$ and $M^{\prime}$ are also equivariantly diffeomorphic. Indeed, we first blow up the fixed point of index 2 on both of these spaces (which has orbifold structure group $\mathbb{Z}_{a+b}$ ) using a weighted blow up of the type described in Section 4.I, corresponding to the circle action on $\mathbb{C}^{2}$ given by

$$
e^{i \theta} \cdot(z, w)=\left(e^{i \theta} z, e^{i(a+b-1) \theta} w\right) .
$$

Note that $\mathbb{Z}_{a+b}$ acts on a neighborhood of this point by

$$
\xi_{a+b} \cdot(z, w)=\left(\xi_{a+b} z, \xi_{a+b}^{-1} w\right)
$$

where $\xi_{a+b}$ is a primitive $(a+b)$-th root of unity. The exceptional divisor will then be a weighted projective space $\mathbb{C} P^{2}(1, a+b-1)$, implying that the blown-up orbifolds $\tilde{M}$ and $\tilde{M}^{\prime}$ will have a singularity of order $a+b-1$. Note that now $\mathbb{Z}_{a+b-1}$ acts on a neighborhood of this point by

$$
\xi_{a+b-1} \cdot(z, w)=\left(\xi_{a+b-1} z, \xi_{a+b-1}^{-(a+b)} w\right)=\left(\xi_{a+b-1} z, \xi_{a+b-1}^{-1} w\right),
$$

where $\xi_{a+b-1}$ is a primitive $(a+b-1)$-th root of unity. Blowing up this singularity in the same way, we obtain a singularity of order $a+b-2$. Continuing to blow up these singularities in this way, we decrease the orders of their orbifold structure groups at each step, until we get two orbifolds equipped with a Hamiltonian $S^{1}$ action having a minimum at an orbifold singularity of order $a$, a maximum at an orbifold singularity of order $b$, and $a+b$ regular index-2 fixed points (cf. Figure 2). If $a=b=1$, then we are done. If not, we blow up the orbifold singularities of index 0 and 4 , using the standard diagonal circle action on $\mathbb{C}^{2}$ given by

$$
e^{i \theta} \cdot(z, w)=\left(e^{i \theta} z, e^{i \theta} w\right) .
$$

The exceptional divisors will now be two smooth spheres which will be fixed by the resulting circle action (cf. Figure 3). This $S^{1}$-action extends to a Hamiltonian action of a 2-torus (cf. $[\mathrm{A}]$ and $[\mathrm{K}]$ ), implying that both $M$ and $M^{\prime}$ can be obtained from a toric manifold by a sequence of weighted blow-downs (and so they are toric orbifolds). As they both have three (isolated) fixed points and the orders of their orbifold structure groups are the same, we conclude that they are equivariantly diffeomorphic (cf. [L-T]). Repeating this argument for spaces with the fixed-point data of Figure 1( $A$ ) (cf. Figure 4), the result follows. 


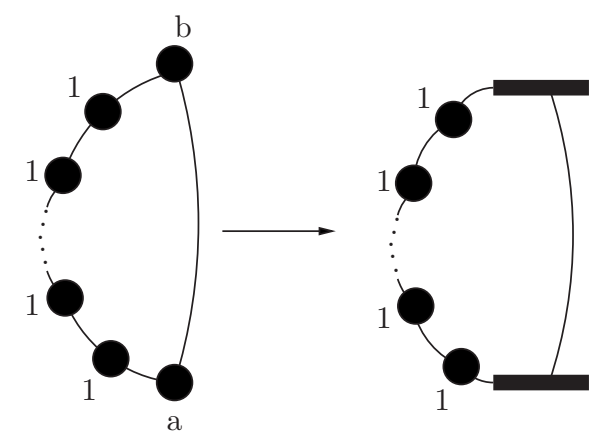

Figure 3. Blowing up fixed points of index 0 and 4

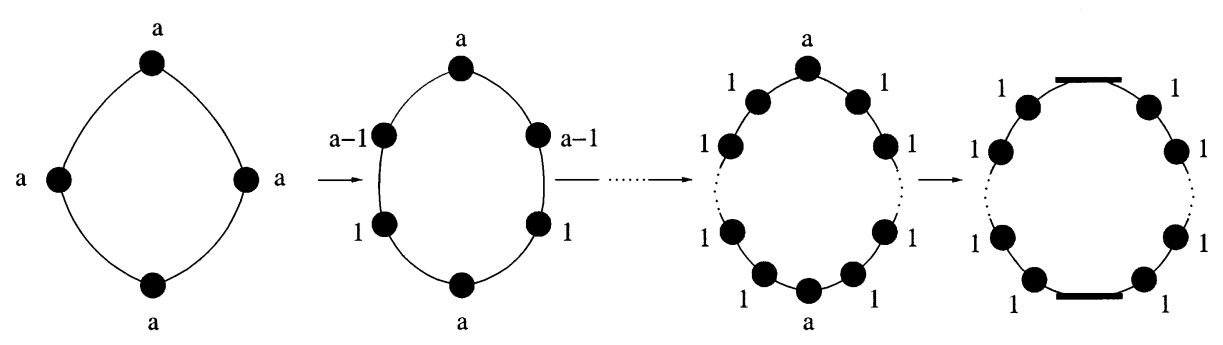

Figure 4. Blowing up fixed points

\section{SOME COMMENTS ABOUT NON-ISOLATED ORBIFOLD SINGULARITIES}

In the previous sections we restricted ourselves, for simplicity, to orbifolds with isolated cone singularities. However, for general cone singularities, we can still blow down gradient "spheres" between index-2 fixed points (using the opposite process of a weighted blow-up similar to the one described in Section 4.I) and obtain an orbifold where all such fixed points are directly connected to both the fixed point of index 0 and the fixed point of index 4 by gradient "spheres". As in the isolated case, we can also blow down some of these spheres ( using the opposite process of a weighted blow-up similar to the one described in Section 4.II), obtaining orbifolds with one or two index-2 fixed points. In the case where there are two of these points, we are in the situation depicted in Figure 5 $(A)$ and then, Proposition 2.1 applied to $\alpha=1$, yields

$$
\frac{d_{0}}{m_{0,1} m_{0,2}}+\frac{d_{4}}{m_{4,1} m_{4,2}}=\frac{d_{1}}{m_{0,1} m_{4,1}}+\frac{d_{2}}{m_{0,2} m_{4,2}} .
$$

Moreover, since we are assuming that we cannot blow down any other gradient "sphere", (6.1) implies that

$$
d_{0}=k m_{0,1} m_{0,2}, \quad d_{1}=k m_{4,1} m_{0,1}, \quad d_{2}=k m_{4,2} m_{0,2}, \quad d_{4}=k m_{4,1} m_{4,2},
$$

for some positive integer $k$. Hence, we have the same fixed-point data (that is, number of fixed points, orders of their orbifold structure groups and their isotropy weights) as in the quotient of the product of two orbifold spheres (teardrops or footballs),

$$
\left(\mathbb{C} P^{1}\left(m_{0,1}, m_{4,2}\right) \times \mathbb{C} P^{1}\left(m_{0,2}, m_{4,1}\right)\right) / \mathbb{Z}_{k},
$$




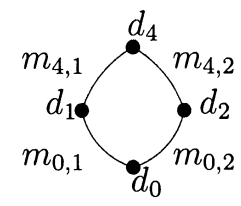

$(A)$

$$
\begin{aligned}
& \left(m_{0,1}, m_{0,2}\right)=1 \\
& \left(m_{4,1}, m_{4,2}\right)=1 \\
& \left(m_{0,1}, m_{4,1}\right)=1 \\
& \left(m_{4,2}, m_{0,2}\right)=1 \\
& \left(m_{0,4}, m_{0,1}\right)=1
\end{aligned}
$$

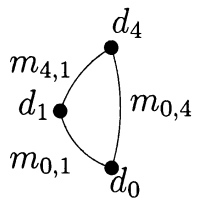

$(B)$

FiguRE 5 .

by the action of $\mathbb{Z}_{k}$ as a subgroup of the circle, acting on the product of the two "spheres" by

$$
e^{\theta i} \cdot\left(\left[z_{1}: z_{2}\right],\left[w_{1}: w_{2}\right]\right)=\left(\left[e^{q \theta i} z_{1}: e^{p \theta i} z_{2}\right],\left[e^{s \theta i} w_{1}: e^{r \theta i} w_{2}\right]\right),
$$

where $p, q, r, s$ are integers satisfying

$$
p m_{0,1}-q m_{4,2}=1 \quad \text { and } \quad r m_{0,2}-s m_{4,1}=1,
$$

equipped with the semifree $S^{1} \cong S^{1} / \mathbb{Z}_{k}$ action induced by that circle action.

In the case where there is only one index-2 fixed point, we are in the situation depicted in Figure 5(B) and, Proposition 2.1 applied to $\alpha=1$, yields

$$
d_{0} m_{4,1}+d_{4} m_{0,1}=d_{1} m_{0,4},
$$

implying that we have the same fixed-point data as in the quotient of a weighted projective space,

$$
\mathbb{C} P^{2}\left(d_{0} / k, d_{4} / k, d_{1} / k\right) / \mathbb{Z}_{k},
$$

with $k=\left(d_{0}, d_{4}\right) / m_{0,4}$ and $d_{1}=\left(d_{0} m_{4,1}+d_{4} m_{0,1}\right) / m_{0,4}$, for an action of $\mathbb{Z}_{k}$ as a subgroup of the circle, acting (on the weighted projective space) as

$$
e^{\theta i} \cdot\left[z_{1}: z_{2}: z_{3}\right]=\left[e^{p \theta i} z_{1}: e^{q \theta i} z_{2}: e^{r \theta i} z_{3}\right],
$$

for integers $p, q, r$ satisfying

$$
q\left(d_{0} /\left(k m_{0,4}\right)\right)-p\left(d_{4} /\left(k m_{0,4}\right)\right)=1 \quad \text { and } \quad p m_{4,1}+q m_{0,1}=r m_{0,4},
$$

equipped with the semifree $S^{1} \cong S^{1} / \mathbb{Z}_{k}$ action induced by that circle action. These integers must exist in all spaces with this fixed-point data in order to have a welldefined semifree circle action on the neighborhood of the gradient sphere connecting the fixed points of index 0 and 4 .

Note that, if we do not assume the orbifold singularities to be isolated, we may not be able to perform a sequence of blow-ups to obtain a manifold as we did in the isolated case. Consequently, we cannot use the classification of $S^{1}$ actions on 4-manifolds, and we can only conclude that any semifree circle action on an orbifold with cone singularities can be obtained by a sequence of weighted blow-ups from a space with the same fixed-point data as one of the above two examples.

\section{ACKNOWLEDGEMENTS}

I am grateful to Michèle Audin for her comments on an earlier version of this work and to the referee whose comments and suggestions helped to improve this paper. 


\section{REFERENCES}

[A] M. Audin, The topology of torus actions on symplectic manifolds, Progress in Mathematics, 93 Birkäuser, Basel, 1991. MR:1106194 (92m:57046)

[A-B] M. F. Atiyah and R. Bott, The moment map and equivariant cohomology, Topology 23 (1984) no. 1, 1-28. MR0721448(85e:58041)

[A-H] K. Ahara and A. Hattori, 4-dimensional symplectic $S^{1}$-manifolds admitting moment map, J. Fac. Sci. Univ. Tokyo, Sect. IA, Math. 38 (1991), 251-298. MR1127083 (93b:58048)

[B-V] N. Berline and M. Vergne, Classes caractéristiques équivariantes. Formule de localisation en cohomologie équivariante, C. R. Acad. Sci. Paris Sir. I Math. 295 (1982), no. 9, 539-541. MR0685019 (83m:58002)

[L-T] E. Lerman and S. Tolman, Hamiltonian torus actions on symplectic orbifolds and toric varieties, Trans. AMS 349 (1997), 4201-4230. MR1401525 (98a:57043)

[K] Y. Karshon, Periodic Hamiltonian flows on four dimensional manifolds, Mem. Amer. Math Soc. 141 (1999). MR.1612833 (2000c:53113)

[M] E. Meinrenken, Symplectic surgery and the Spin ${ }^{c}$-Dirac operator, Advances in Mathematics 134 (1998), 240-277. MR1617809 (99h:58179)

[MD] D. McDuff, The moment map for circle actions on symplectic manifolds, Journal of Geometry and Physics 5 (1998), no. 2, 149-160. MR.1029424 (91c:58042)

[R] S. Roan, Picard groups of hypersurfaces in toric varieties, Publ. RIMS, Kyoto Univ. 32 (1996), 797-834. MR1433319 (98f:14003)

[T-W] S. Tolman and J. Weitsman, On semifree symplectic circle actions with isolated fixed points, Topology 39 (2000), 299-309. MR.1722020(2000k:53074)

Departamento de Matemática, Instituto Superior Técnico, Av. Rovisco Pais, 1049001 Lisbon, Portugal

E-mail address: lgodin@math.ist.utl.pt 